\title{
MULTISPECTRAL MEDICAL IMAGE FUSION FOR ALZHEIMER DISEASE USING ADAPTIVE BLOOD FLOW WEIGHT BASED FUSION RULE
}

\author{
D. Sheefa Ruby Grace ${ }^{1 \S}$, Mary Immaculate Sheela ${ }^{2}$ \\ ${ }^{1}$ Bharathiar University \\ Coimbatore, INDIA \\ ${ }^{2}$ Pentecost University \\ Accra, GHANA
}

\begin{abstract}
Nowadays medicinal treatment is highly depending on medical images. There are variety of multi modal medical images are available. Each images are having certain pros and cons. Hence, there is a need of image fusion of multiple images to get a single informative image. In this paper, SPECT (Single Photon Emission Computed Tomography) and MRI (Magnetic Resonance Image) images are taken as source images. They are the images of Alzheimer disease affected patients. In this proposed method, the Adaptive Blood Flow Weight, $\mathrm{ABF}_{\text {WEIGHT }}$ for each image is calculated based on the severity of the disease. The blocks of each image is applied contourlet transform, so that it yields Low Frequency Coefficients (LFC) and High Frequency Coefficients (HFC) separately. The High Frequency coefficients are fused using Guided Filter algorithm. The Lower Frequency coefficients are fused using the proposed Adaptive Blood Flow (ABF) fusion rule. Finally inverse transform is applied to get fused image. The work is implemented in MATLAB. It has been proved that the proposed method provides better result. The quality of fused images are computed using the performance measures like entropy, Peak Signal to Noise Ratio (PSNR), Edge strength (Q) and Fusion Factor (FF).
\end{abstract}

AMS Subject Classification: $68 \mathrm{U} 10$

Key Words: adaptive blood flow, Alzheimer, contourlet transform, guided filter

Received: June 16, 2018

(C) 2018 Academic Publications

${ }^{\S}$ Correspondence author 


\section{Introduction}

Image fusion is a process of merging two or more images which are acquired by different sensors into a sole image. The resultant fused image contains important features of source images which helps physicians to diagnose diseases accurately and to offer treatment perfect [7]. Since the diagnosis is dependent on the medical images, it should be carefully fused. A good image fusion method should have the following properties. First, it should preserve most of the useful information of different images needed for further processing. Second, it should not produce artifacts in fused image. Third, it must be robust to lacking conditions such as mis-registration and noise [13].

Alzheimer is a disease of brain disorder. Severe Alzheimer will affect the functioning of brain. It is very difficult to restrict the disparaging process of disease. Even though the Alzheimer disease is not curable, if it is diagnosed in the early stage, the spreading of the disease can be slow down [14]. Hence, computer aided diagnosis is required with good fusion method. The proposed method uses contourlet based guided filter method with $\mathrm{ABF}_{\text {WEIGHT }}$ estimation method, for the multispectral images of Alzheimer disease.

SPECT image is known as multispectral image whereas MRI image is known as panchromatic image. MRI image provides the accurate structural information of brain. It helps to improve the treatments so that the progress of degeneration in Alzheimer disease is well done. SPECT image captures functional information from the source image which helps in diagnosing the disease perfectly and testing the drugs given. Hence, the proposed method fuses the SPECT and MRI image to get useful information from both source images.

Several image fusion methods are available in medical imaging field of research. The image fusion methods can be broadly classified into two groups - spatial domain fusion and transform domain fusion. The spatial domain fusion approaches include averaging method, select maximum, select minimum method, Brovey method, Principal Component Analysis (PCA), Intensity-Hue Saturation (IHS) based methods and high pass filtering based techniques. The disadvantage of spatial domain approaches is that, they produce spatial distortions in the fused image. The spatial distortions are well handled by transform domain approaches such as Laplacian pyramid based transform, Discrete wavelet based transform, Curvelet based transform and Contourlet based transform etc.

In recent years, multi-resolution transforms have been recognized as a very useful approach to analyze the information content of images for the purpose of image fusion [1]. In this paper, a multi-resolution contourlet transform has 
been used to obtain the coefficients of the blocks of source images.Before implementing the proposed method, different already existing methods that were proposed by various researchers were thoroughly analyzed.

Anuyogam et al. [2] proposed an image fusion algorithm using the hybrid of Principal Component Analysis (PCA) and Orthogonal Matching Pursuit (OMP). The weighted average fusion is used to fuse the sparse PCA result with the common feature thereby preserving the edge information and high spatial resolution. Om Prakash et al. [10] proposed a pixel-level image fusion scheme using multiresolution steerable pyramid wavelet transform. Wavelet transform has been applied at different decomposition levels. Then the coefficients are fused using maximum fusion rule. The advantages of steerable pyramid wavelet transform are shift invariance and selfreversibility. They can preserve edge, lines, curves, boundaries information and the distortion in the fused image has been reduced. Sudeb et al. [12] implemented a novel multi modal medical image fusion algorithm employing multiscale geometric analysis of non-subsampled contourlet transform and fuzzy-adaptive reduced pulse-coupled neural network (RPCNN). Use of RPCNN with less complex structure and having less number of parameters, leads to computational efficiency. Hima et al. [5] proposed a multi modal image fusion method using content based automatic segmentation. Images are segmented into regions using automatic segmentation process and they are fused according to region based fusion rule. The Region Correlation Coefficient is calculated for all the regions so that the best region is chosen to construct the fused image.

Shutao et al. [13] has presented a fast two-scale fusion method. Guided filtering is used as local filtering method for image fusion. The parameters of guided filter is used to control pixel saliency and spatial consistency measures. However, the drawback is the guided image filter cannot represent the some edges of the image well and the weight map construction method also not represented the visual features in source images. Vikrant et al. [14] proposed a multimodal medical image fusion based on directive contrast in NSCT domain. Phase congruency method is used to fuse low frequency coefficients whereas directive contrast method is used for fusing high frequency coefficients of source images. Yong et al. [16] proposed a multisensory image fusion method by using gradient domain guided filtering with multiple visual features measurement. A decision map construction model has been presented. The key factors of this model are contrast saliency, sharpness and structural saliency which improved the performance of fusion result. Despite the parameters are not chosen according to the different types of source images. Gaurav et al. [3] designed a novel fusion framework multimodal medical images based on non-subsampled 
contourlet transform (NSCT). Medical images are used as source images. First, the source images are transformed by NSCT so that the low frequency components and high frequency components are produced. Then the coefficients are combined. Low frequency components are fused by phase congruency fusion rule whereas the high frequency components are fused by directive contrast fusion rule.

Since all the already existing methods have some drawbacks, a good fusion method is necessary to overwhelm the downsides. In this proposed method, the high frequency coefficients of both SPECT and MRI images are fused using contourlet based Guided Filter method. Low frequency coefficients are fused by the proposed $\mathrm{ABF}_{\text {WEIGHT }}$ estimation method. Finally, inverse transform is applied to get the fused image.

\section{Methods}

In the proposed method, multispectral image SPECT and a multimodal image MRI are used as source images. MRI is monochrome image whereas SPECT is a color image. Applying transforms directly to the color space of SPECT image will alter the color balance and loss of information. We cannot identify the specific color and objects properly. So, the Red (R), Green (G) and Blue (B) channel coefficients of SPECT image is separated. Then, the Adaptive Blood Flow Weight, $\mathrm{ABF}_{\text {WEIGHT }}$ is calculated for each image using the Red $(\mathrm{R})$ channel of SPECT image. The source images are divided into non-overlapping blocks, each of size $m \times n$. Now, multi resolution contourlet transform is applied to both blocks of the source images. After applying the contourlet transform, the low frequency coefficients and the high frequency coefficients of both SPECT and MRI are separated. The low frequency coefficients are fused using the proposed Adaptive Blood Flow weight method which used the so far calculated $\mathrm{ABF}_{\text {WEIGHT. }}$. The high frequency coefficients of the blocks of both SPECT and MRI images are fused by guided filter method. Finally, inverse contourlet transform is applied to get fused image.

\section{Calculation of Adaptive Blood Flow Weight, ABFweight:}

The block based Adaptive Blood Flow Weight, ABF WEIGHT is calculated using Red ( R) channel of SPECT image. SPECT scan produces images that show how your organs work. A SPECT scan can show how blood flows to your heart or what areas of your brain are more active or less active. The Red color in SPECT image shows the severity of the disease. The most affected part is usually denoted by the color Red. Hence, ABF WEIGHT is calculated according 
to the ABF_THRESH value of the $R$ channel coefficient of SPECT image, say $R_{\text {SPECT }}$. The following equation (1) is used to calculate $A B F_{\text {WEIGHT }}$

$$
\begin{aligned}
& \text { If } R_{\mathrm{SPECT}}(i, j) \begin{cases}W_{\mathrm{MRI}}(i, j)=0.7, & \text { if }>A B F \_T H R E S H, \\
W_{\mathrm{MRI}}(i, j)=0.5, & \text { otherwise, }\end{cases} \\
& W_{\mathrm{SPECT}}(i, j)=1-W_{\mathrm{MRI}}(i, j) .
\end{aligned}
$$

\section{Contourlet Transform:}

The source images SPECT and MRI are divided into non-overlapping blocks of size $m x n$. Then the blocks are decomposed using frequency domain image fusion method, the contourlet transform. Comparing to wavelet transform, contourlet transform deals with the curve like edges accurately.

The general contourlet transform uses the Laplacian pyramid (LP) decomposition followed by a Directional Filter Bank (DFB). At each decomposition level, the LP decomposes the image into different scale of low pass and high pass sub bands and DFB is applied to the decomposed images for filtering the bands [6]. Hence, each blocks are decomposed into Low Frequency Coefficients, say LFC $_{\text {SPECTblk }}$, LFC $_{\text {MRIblk }}$ and High Frequency Coefficients, say $\mathrm{HFC}_{\text {SPECTblk }}, \mathrm{HFC}_{\text {MRIblk}}$.

Fusion of Low Frequency Coefficients by Adaptive Blood Flow (ABF) Fusion Rule:

After decomposition, fusion rule is applied in two levels. The high frequency coefficients (HFC) are fused using guided filtering method. The low frequency coefficients (LFC) are fused using Adaptive Blood Flow (ABF) method using the calculated $\mathrm{ABF}_{\text {WEIGHT }}$. The LFC of SPECT block and MRI block is $\mathrm{LFC}_{\text {SPECTblk }}$ and $\mathrm{LFC}_{\text {MRIblk }}$ respectively. The fused LFC of a SPECT block is obtained by multiplying the average values of $\mathrm{ABF}_{\text {WEIGHT }}$ of SPECT block, say $W_{\text {SPECT }}$ by LFC coefficients SPECT block. Similarly, the fused LFC of MRI block is obtained by multiplying the average values of ABF WEIGHT of MRI, say $\mathrm{W}_{\text {MRI }}$ by LFC coefficients MRI block. Summation of the two values yields the fused LFC block, FUSED LFCBLK. The following equation (2) is used to calculate the coefficients of FUSED LFCBLK $_{\text {. }}$

\section{Fusion of High Frequency Coefficients by Guided Filter Method (GF):}

Guided Filter (GF) Method is an edge-preserving filter method. It can avoid artifacts and provide high contrasted strong edges of the image. The general GF process is using an input image, a guidance image and an output image. Sometimes the guidance image may be the input image itself. The 
GF provides a linear transform of the guidance image as output [9]. In this proposed method, GF is used to fuse high frequency coefficients of SPECT and MRI images such as

FUSED $_{\text {HFCBLK }}=\mathrm{GF}\left(\right.$ HFC $_{\text {SPECTblk }}$, HFC $\left._{\text {MRIblk }}\right)$.

Finally inverse contourlet transform is applied and hence a fused image is generated. The stepwise algorithm is given as follows:

Input: Two input images SPECT and MRI of same size,

Output: A single fused image, FI .

1. Read the two different multi-modal medical images, SPECT and MRI to be fused.

2. MRI is monochrome image whereas SPECT is color image

3. Separate the Red (R), Green (G), Blue (B) channel coefficients of the SPECT image

4. Calculate block based Adaptive Blood Flow Weight, $\mathrm{ABF}_{\text {WEIGHT }}$ for each image

5 .

using the Red (R) channel coefficient of SPECT image

If $R_{\mathrm{SPECT}}(i, j) \begin{cases}W_{\mathrm{MRI}}(i, j)=0.7, & \text { if }>A B F_{-} T H R E S H \\ W_{\mathrm{MRI}}(i, j)=0.5, & \text { otherwise, }\end{cases}$

$W_{\mathrm{SPECT}}(i, j)=1-W_{\mathrm{MRI}}(i, j)$.

6. Divide both input images into non-overlapping blocks of size $m x n$,

7. Apply the contourlet transform to each block of both multi-modal images,

8. Each blocks are decomposed into

i) Low Frequency Coefficients, say $\mathrm{LFC}_{\mathrm{SPECTblk}}, \mathrm{LFC}_{\mathrm{MRIblk}}$,

ii) High Frequency Coefficients, say $\mathrm{HFC}_{\mathrm{SPECTblk}}, \mathrm{HFC}_{\mathrm{MRIblk}}$,

9. Apply fusion rule for Low Frequency Coefficients based on ABF WEIGHT, 
10. Apply Guided Filter fusion rule for High Frequency Coefficients as $\mathrm{FUSED}_{\text {HFCBLK }}=\mathrm{GF}\left(\mathrm{HFC}_{\text {SPECTblk }}, \mathrm{HFC}_{\text {MRIblk }}\right)$,

11. Apply Inverse Transform to get the fused block FUSEDblk

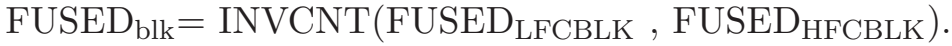

The outline of the proposed method is given in Figure 1.

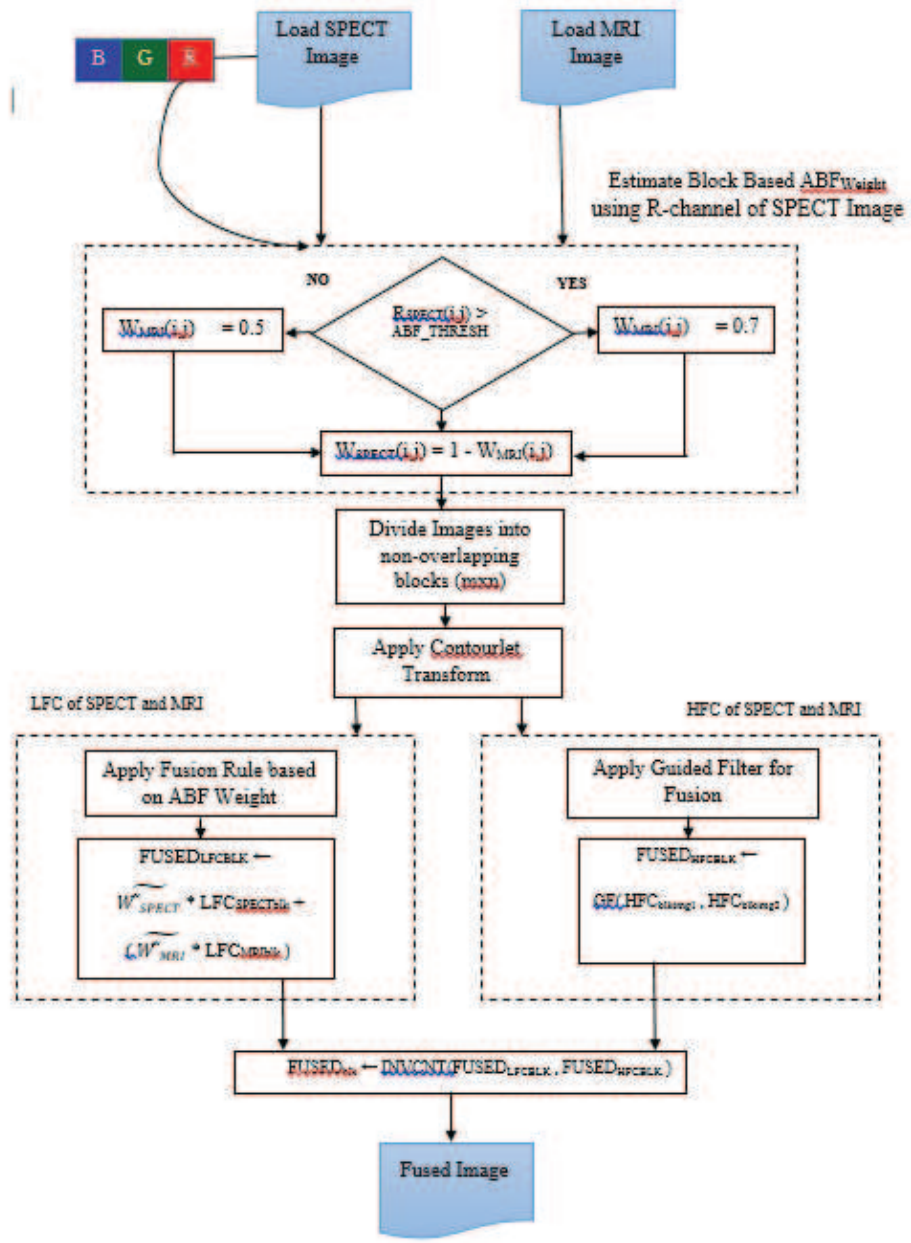

Figurel Outline of the Proposed Method

Figure 1: Outline of the Proposed Method 


\section{Results}

The general requirement of an image fusing process is to preserve all valid and useful information from the source images, while at the same time it should not introduce any distortion in resultant fused image. Performance measures are used to measure the possible benefits of fusion method.

\section{Performance Metrics:}

Performance measures are used to evaluate the quality of the fused image. The fused images are evaluated, by taking the following parameters into consideration. From the literature survey, it is evident that the performance metrics such as Entropy (EN), Peak Signal to Noise Ratio (PSNR), Structural Similarity Index Measure (SSIM),Fusion Factor (FF) and Edge Strength (Q) are the effective quality measures. The higher values represent the high quality of fused image [8].

\section{Entropy (EN):}

One of the quantitative measures used in digital image processing is entropy, introduced by Claude Shannon, the entropy concept was first utilized in order to quantify the information content of messages. Larger alterations and changes in an image give larger entropy values and sharp, focused images have more changes than blurred and misrouted images

$$
E N=\sum_{l=0}^{L-1} p(l) \log 2 p(l),
$$

where $p(l)$ is the probability of gray level $l$. The fused image which contain the maximum of Entropy will give the best fusion technique.

\section{Peak Signal to Noise Ratio (PSNR):}

The PSNR is used to calculate the similarity between two images. The PSNR between the reference image $R$ and the fused image $F$ is calculated as

$$
P S N R=10 \times \log \left(\frac{f \max ^{2}}{M S E^{2}}\right),
$$

where $f \max$ is the maximum gray scale value of the pixels in the fused image. Higher the value of the PSNR, better the performance of the fusion algorithm.

\section{Fusion Factor $(F F)$ :}

Given two images $A$ and $B$, and their fused image $F$, the Fusion factor $(F F)$ is

$$
F F=I_{A F}+I_{B F},
$$


where $\mathrm{I}_{\mathrm{AF}}$ and $\mathrm{I}_{\mathrm{BF}}$ are the MIM values between input images and fused image. A higher value of FF indicates that fused image contains moderately good amount of information present in both the images.

\section{Edge Strength $(Q)$ :}

For two source images $A, B$ and fused image $F$, the Edge Strength $Q$ is defined as

$$
Q=\frac{\sum_{n=1}^{N} \sum_{m=1}^{M} Q^{A F}(n, m) W^{A}(n, m)+Q^{B F}(n, m) W^{B}(n, m)}{\sum_{i=1}^{N} \sum_{j=1}^{M}\left(W^{A}(i, j)+W^{B}(i, j)\right)},
$$

where $Q^{\mathrm{AF}}(n, m)$ and $Q^{\mathrm{BF}}(n, m)$ are edge information preservation values. The value of $Q$ ranges in between 0 and 1. Value 0 denotes the complete loss of edge information and 1 indicates fusion with no loss of edge information. Since metric represents the edge information associated with the fused image and visually supported by human visual system. Hence a higher value of $Q$ value implies better edge preserved fused image.

\section{Discussion}

The source images used in this paper are retrieved from the Harvard University Database. In this paper, seven number of image sets are considered for analysis. All the source images are Alzheimer disease affected images. This work has been implemented using MATLAB.

\section{Case \#1:}

The first case was a 73 year old woman, who was suffering from Alzheimer disease because of a 3 year history of memory loss. She had completed high school and worked in a clerical position until her retirement. She had lived alone and maintained her own home and financial affairs since the death of her husband. The neighbors had begun to notice gradually worsening memory impairment and difficulty finding words, but she had no complaints. She was a well-groomed woman who was alert and friendly. General and elemental neurological exams were normal.

For this case \#1, the MRI and SPECT scan have been taken at different time period, usually in months. Image set1, Image set4, Image set5 and Image set6 are belongs to case1. The source images are MR-T2 and SPECT-Tc and the MRI image shows many features of the disease whereas SPECT image provides functional information. The fused image which is obtained by the proposed 
method provides both the information and it is given in Figure 2, Figure 3, Figure 4 and Figure 5 for the Image set1, Image set4, Image set5 and Image set6 respectively.

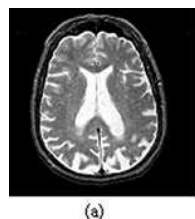

(a)

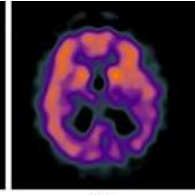

(b)

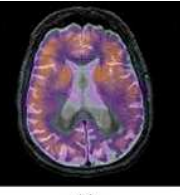

(c)

Figure 2: Fusion Result of Image Set 1 (a) MR-T2 (b) SPECT-Tc (c) Fused Image

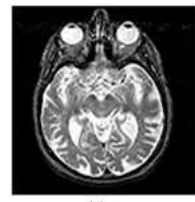

(a)

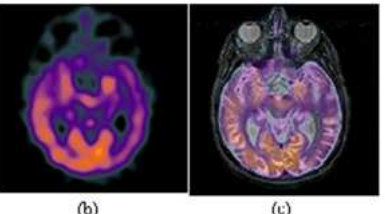

(c)

Figure 3: Fusion Result of Image Set 4 (a) MR-T2 (b) SPECT-Tc (c) Fused Image

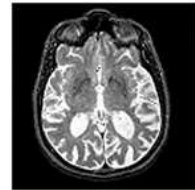

(a)

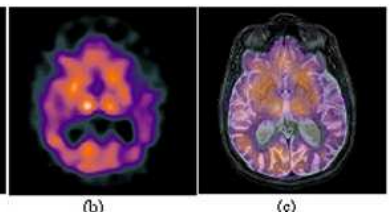

(b)

(c)

Figure 4: Fusion Result of Image Set 5 (a) MR-T2 (b) SPECT-Tc (c) Fused Image

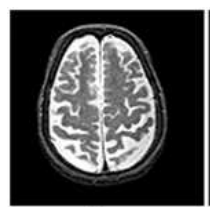

(a)

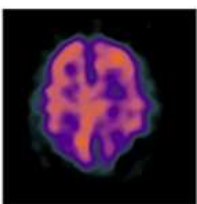

(b)

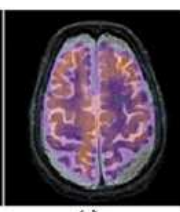

(c)

Figure 5: Fusion Result of Image Set 6 (a) MR-T2 (b) SPECT-Tc (c) Fused Image

\section{Case \#2:}

The second case was a 74 year old woman who was healthy and took no medications She developed difficulty with recalling recent events, names, and appointments. The family reported slow, gradual progression over 2 years. She 
was able to care for her ailing husband, and required only occasional assistance from family members in keeping track of financial affairs.

For this case\# 2, both MRI and SPECT scan have been taken at different time period, usually days. Image set2 and Image set7 consists of MR-PD and SPECT-Tc as source images. Image set3 has MR-T1 and SPECT-Tc as source images. MR images show a mild degree of cerebral atrophy, as slight widening of the hemispheric sulci, prominent in the parietal and perisylvian regions. Perfusion SPECT is abnormal in a pattern consistent Alzheimer's disease. The fused image which is obtained by the proposed method provides both the information and it is given in Figure 6, Figure 7 and Figure 8 for the Image set2, Image set7 and Image set3 respectively.

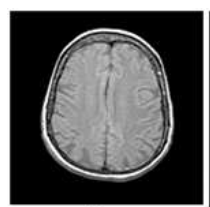

(a)

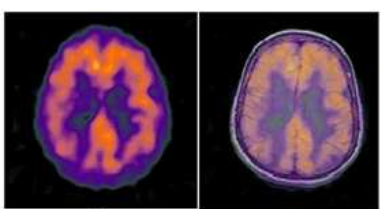

(c)

Figure 6: Fusion Result of Image Set 2 (a) MR-PD (b) SPECT-Tc (c) Fused Image

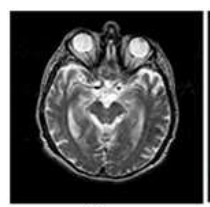

(a)

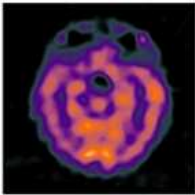

(b)

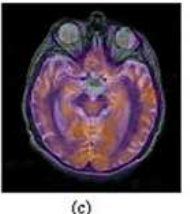

(c)

Figure 7: Fusion Result of Image Set 7 (a) MR-PD (b) SPECT-Tc (c) Fused Image

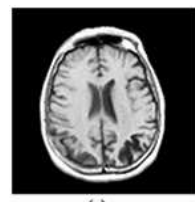

(a)

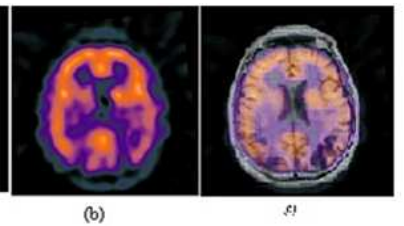

(b)

Figure 8: Fusion Result of Image Set 3 (a) MR-T1 (b) SPECT-Tc (c) Fused Image

The quality of fused images are analyzed using the proposed method for various performance measures and the values are summarized in Table 1.

Moreover, the Image set1, Image set2 and Image set3 are analyzed and the achieved results are compared with the already existing medical image fusion 


\begin{tabular}{l|l|l|l|l|l|l}
\hline \multicolumn{5}{c|}{ ENTROPY } & \multicolumn{3}{l}{} \\
\hline $\begin{array}{l}\text { Image } \\
\text { Set }\end{array}$ & MRI & SPECT & Fused & PSNR & FF & Q \\
\hline Set 1 & 3.49 & 4.27 & 5.32 & 68.92 & 6.01 & 0.74 \\
Set 2 & 3.28 & 4.33 & 4.93 & 64.42 & 5.73 & 0.74 \\
Set 3 & 3.26 & 4.75 & 5.61 & 67.70 & 6.57 & 0.73 \\
Set 4 & 3.71 & 4.34 & 5.93 & 68.34 & 6.18 & 0.76 \\
Set 5 & 3.63 & 4.38 & 5.42 & 69.44 & 5.98 & 0.74 \\
Set 6 & 3.21 & 3.70 & 4.70 & 60.45 & 5.54 & 0.73 \\
Set 7 & 3.93 & 4.56 & 5.93 & 62.78 & 5.58 & 0.73 \\
\hline
\end{tabular}

Table 1: Performance Analysis of Proposed Method for Various Measures

methods such as Wavelet, Curvelet, Contourlet and NSCT with the proposed $\mathrm{ABF}_{\text {WEIGHT }}$ fusion method [14]. The various performance measures such as Entropy, PSNR, Q and FF are used to compare the quality of resultant fused image quantitatively. The values are summarized in Table 2 .

Vikrant et al. [14] has implemented a Non Sub-sampled Contourlet Transform (NSCT) based fusion method and compared their result with the various fusion methods such as Wavelet, Curvelet and Contourlet. It has been proved that NSCT method provided the better fusion result. In this paper, the proposed $\mathrm{ABF}_{\text {WEIGHT }}$ fusion method has been compared with the NSCT method and it provides the best result. Figure 9 shows the visual output of source and fused images.

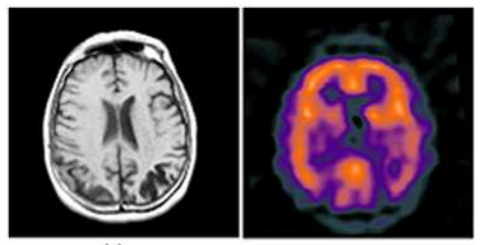

(a)

(b)
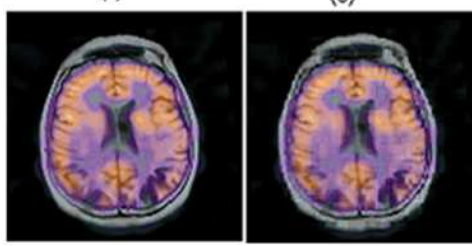

(c)

(d) 


\begin{tabular}{|c|c|c|c|c|c|}
\hline Image Set & Fusion Methods & EN & PSNR & Q & FF \\
\hline \multirow{5}{*}{ Set1 } & Wavelet & 4.38 & 64.96 & 0.66 & 2.88 \\
& Curvelet & 4.32 & 62.21 & 0.66 & 3.04 \\
& Contourlet & 4.65 & 67.62 & 0.67 & 3.22 \\
& NSCT & 5.15 & 68.30 & 0.70 & 3.31 \\
& Proposed & 5.32 & 68.92 & 0.74 & 6.01 \\
\hline \hline \multirow{5}{*}{ Set2 } & Wavelet & 3.91 & 62.11 & 0.58 & 2.92 \\
& Curvelet & 3.95 & 64.12 & 0.60 & 3.51 \\
& Contourlet & 4.65 & 65.49 & 0.60 & 3.21 \\
& NSCT & 4.76 & 65.49 & 0.66 & 3.76 \\
& Proposed & 5.61 & 67.70 & 0.73 & 6.57 \\
\hline \hline \multirow{5}{*}{ Set3 } & Wavelet & 5.74 & 65.32 & 0.51 & 4.54 \\
& Curvelet & 5.83 & 64.92 & 0.48 & 3.51 \\
& Contourlet & 5.74 & 64.10 & 0.51 & 2.41 \\
& NSCT & 5.89 & 67.33 & 0.65 & 4.77 \\
& Proposed & 5.93 & 68.34 & 0.76 & 6.18 \\
\hline
\end{tabular}

Table 2: Comparison of the Performance Values of Proposed Method with Various Fusion Methods

Figure 9: Fusion Result of Image Set 3 (a) MR-T1 (b) SPECT-Tc

(c) Fused Image by NSCT3 (d) Fused Image by Proposed method

\section{Conclusion}

In this paper, multispectral medical image fusion method has been proposed for fusing the Alzheimer disease affected MRI and SPECT images. Adaptive Blood Flow weight has been estimated based on the Red Channel of SPECT image. Contourlet transform is applied to separate the Low Frequency and High Frequency coefficients of both MRI and SPECT. Adaptive Blood Flow Weight estimation method is used to fuse LFC of MRI and SPECT whereas Guided Filter method is applied to fuse HFC of MRI and SPECT. The quality of the fused image is measured using various performance measures such as Entropy, PSNR, FF, SSIM and $Q$. The result shows the proposed method gives better result comparing to other existing methods. 


\section{References}

[1] T.S. Anand, K. Narasimhan, P. Saravanan, Performance evaluation of image fusion using the multi-wavelet and curvelet transforms, IEEE International Conference on Advances in Engineering, Science and Management, 12 (2012).

[2] A. Venkataraman, J. Alirezaie, P. Babyn, A. Ahmadian, Medical image fusion based on joint sparse method, IEEE Conference on Biomedical Engineering (MECBME), 14 (February 17-20, 2014), Hilton Hotel, Doha, Qatar.

[3] G. Bhatnagar, J. Wu, Q.M., Z. Liu, Directive contrast based multimodal medical image fusion in NSCTdomain, IEEE Transactions on Multimedia, 13 (2013), Vol. 15, No 5.

[4] G. Yang, M. Li, L. Chen, J. Yu, The nonsubsampled contourlet transform based statistical medical image fusion using generalized Gaussian density, Computational and Mathematical Methods in Medicine, 15 (2015), Article ID 262819, 13 pages, Hindawi Publ. Co.

[5] C.H. Hima Bindu, K. Veera Swamy, Medical image fusion using content based automatic segmentation, IEEE International Conference on Recent Advances and Innovations in Engineering, 14 (May 9-11, 2014), Jaipur, India.

[6] J. Kaur, S. Sharma, S. Gupta, A novel technique for medical image fusion using improved contourlet transformation with modified DFBs, IEEE Conference, 13 (July 4-6, 2013), Tiruchengode, India.

[7] J. Agarwal, S.S. Bedi, Implementation of hybrid image fusion technique for feature enhancement in medical diagnosis, Human-centric Computing and Information Sciences, 5, No 3 (2015), 17 pp; doi: 10.1186/s13673-0140020-z.

[8] K. Kalaivani, Y. Asnath Victy Phamila, Analysis of image fusion techniques based on quality assessment metrics, Indian Journal of Science and Technology 16 (August 2016), Vol. 9 (31).

[9] Z. Liu, H.Yin, Y. Chai, S.X. Yang, A novel approach for multi-modal medical image fusion, Expert Syst. Appl., 14, 41 (16) (2014), 7425-7435. 
[10] Om Prakash, A. Kumar, A. Khare, Pixel-level Image fusion scheme based on steerable pyramid wavelet transform using absolute maximum selection fusion rule, IEEE, 14 (2014), 978-1-4799-2900-9/14.

[11] H. Patel, V. Bhateja, A. Krishn, A. Sahu, Medical image fusion in curvelet domain employing PCA and maximum selection rule, Proc. of the 2nd International Conference on Computers and Communication Technologies, 15 (2015), Hyderabad, India, Springer.

[12] S. Das, M. Kumar Kundu, A neuro-fuzzy approach for medical image fusion, IEEE Transactions on Biomedical Engineering, 13 (2013).

[13] S. Li, X. Kang, J. Hu, Image fusion with guided filtering, IEEE Transactions On Image Processing, 13 (2013), Vol. 22, No 7.

[14] V. Bhateja, A. Moin, A. Srivastava, Le Nguyen Bao, A. Lay-Ekuakille, Dac-Nhuong Le, Multispectral medical image fusion in contourlet domain for computer based diagnosis of Alzheimers disease, American Institute of Physics, Rev. Sci. Instrum., 16 (2016).

[15] L. Yang, B.L. Guo, W. Ni, Multimodality medical image fusion based on multiscale geometric analysis of contourlet transform, Neurocomputing, $\mathbf{0 8}$ (2008), 72, 203211.

[16] Y. Yang, Y. Que, S. Huang, P. Lin, Multiple visual features measurement with gradient domain guided filtering for multisensor image fusion, IEEE Transactions on Instrumentation and Measurement, 17 (2017). 
